The Astrophysical Journal, 191: 173-182, 1974 July 1

(c) 1974. The American Astronomical Society. All rights reserved. Printed in U.S.A.

\title{
THEORETICAL EVOLUTION OF EXTREMELY METAL-POOR STARS
}

\author{
RAYMOND L. WAGNER \\ University of Texas, Austin; and University of Washington, Seattle* \\ Received 1973 October 18: revised 1974 January 14
}

\begin{abstract}
Theoretical evolutionary sequences for stars in the mass range 0.65 to $2.5 M_{\odot}$ having the extremely metaldeficient compositions $(X, Z)=\left(0.739,10^{-4}\right),\left(0.739,10^{-6}\right),\left(0.739,10^{-8}\right),\left(0.9999,10^{-8}\right)$ have been calculated using a modified version of the Paczyński stellar-evolution code. The models have been evolved from the zero-age main sequence through the central density maximum accompanying core helium ignition. Selected models in each evolutionary sequence have been analyzed for secular and pulsational instability. The characteristics of extremely metal-poor stellar models were found to be somewhat sensitive to the heavy-element abundance even below $Z \simeq 10^{-4}$, and low-mass stellar lifetimes from the main sequence to central helium ignition were found to be significantly shorter than values commonly assumed for galactic evolution studies. The main-sequence models of lowest mass are pulsationally unstable with fundamental periods of about 0.67 hour. These models become pulsationally stable before central hydrogen exhaustion. The most massive stellar models for each composition become pulsationally unstable at core helium ignition, with the pulsation periods for the fundamental mode lying in the range $0.3-4.0$ days. Evolutionary tracks and time-constant loci are presented for each composition.
\end{abstract}

Subject headings: interiors, stellar — Population II stars - pulsation - stellar evolution

\section{INTRODUCTION}

It has been evident for more than a decade that there exists a definite relationship between the ages, compositions, and kinematic properties of stars in our Galaxy which can be explained by a model in which the Galaxy rapidly collapsed from a rotating spheroidal cloud of gas into a thin disk of stars and gas (Eggen, Lynden-Bell, and Sandage 1962). In order to explain the very rapid rate of metal enrichment of the infalling gas, and also the apparent absence of surviving members of the initial generation of stars, it has been inferred that the initial stellar population consisted primarily, or even solely, of massive stars which would speed through their nuclear burning stages, become supernovae, and mix their nuclear products into the interstellar medium before the collapse halted (Schmidt 1963; Truran, Hansen, and Cameron 1965; Truran and Cameron 1971). Possible alternatives to a variable initial-mass function have also been suggested, including models with continuing infall of metal-poor primordial material (Larson 1972; Fowler 1972; Quirk and Tinsley 1973) and models with the rate of star formation enhanced by high metal content in the interstellar gas (Talbot 1973; Talbot and Arnett $1973 a, b)$. Although it is possible to formulate the problem of galactic nucleosynthesis in such a way that many potentially observable quantities are independent of the details of the chronological history of the enrichment process (cf. Talbot and Arnett 1971, $1973 a, b)$, treatment of abundance variations with time requires a knowledge of stellar lifetime as a function of initial mass and composition.

The evolutionary calculations reported here were performed in order to provide a uniform set of stellar

* Current address. lifetimes which can be used for low- and intermediatemass initial-population stars during galactic-evolution calculations. The compositions chosen were selected to cover the possible range of initial compositions, with $Z=10^{-4}$ corresponding to the most metaldeficient stars known (Pagel 1970; Greenstein 1970). Values of $Z=10^{-6}$ and $10^{-8}$ were also chosen, bracketing the maximum heavy-element enrichment by processing in supermassive objects or the big bang (Wagoner 1973, and references therein). Since stellar opacities effectively cease to change with decreasing metal content for $Z \leqslant 10^{-6}$, the most metal-poor models correspond to pure hydrogen-helium stars. A sequence of essentially pure hydrogen $(X, Z=0.9999$, $10^{-8}$ ) models was also computed in order to provide data for calculations beginning with pure hydrogen protogalaxies. A typical Population I hydrogen abundance of $X=0.739$ was used for the other model sequences. Stellar models at principal points in each evolutionary sequence were analyzed for secular and pulsational instability.

In $\S$ II below, the evolution and analysis programs are briefly described. Evolutionary sequences and results of the pulsation and stability calculations for each set of models are presented in $\S$ III and $\S$ IV, respectively. These results are discussed in the final section.

\section{COMPUTATIONAL TECHNIQUE}

\section{a) Input Physics}

The evolution program and the stability analysis program both use the same "input physics," which is described in this subsection.

Logarithms of the radiative opacity were interpolated linearly in $Z$ and in $\log X$ from a set of stellar 
opacity tables computed for values of $Z=0$ and $Z=10^{-4}$ and varying values of $X$ by the Los Alamos opacity group (Cox and Stewart 1969, 1970a, $b$; Merts and Magee 1972). Values of opacities interpolated in this way have been compared with Los Alamos calculations for $(X, Z)=\left(0.739,10^{-6}\right)$ and both the standard heavy-element mix and a heavy-element mixture consisting primarily of ${ }^{7} \mathrm{Li}$ with smaller amounts of $B, C, N$, O which corresponds to predicted big-bang production (cf. Wagoner 1973). (These later tables were kindly computed for the author by A. L. Merts and N. H. Magee of Los Alamos.) Differences between the three sets of opacities were much smaller than the estimated accuracy of the Los Alamos computations of 15-30 percent. Conductive opacities for thermal conduction by degenerate electrons were interpolated from the tables of Hubbard and Lampe (1969).

Convective energy transport in the envelope was treated using the standard mixing-length theory (Vitense 1953; Böhm-Vitense 1958) with the ratio of mixing length to pressure scale height taken to be unity. In the interior, regions in convective equilibrium according to the Schwarzschild criterion $\nabla_{\mathrm{ad}}<\nabla_{\mathrm{rad}}$ (Schwarzschild and Härm 1958) were treated as completely mixed. Semiconvection, if it occurred, was treated according to the method of Robertson and Faulkner (1972). Energy losses due to neutrino emission in hydrogen-exhausted regions of the models were interpolated from tabulated data prepared using the expressions of Beaudet, Petrosian, and Salpeter (1967) for the photo-neutrino, plasma-neutrino, and pair annihilation processes. Other possible neutrino processes were ignored, including Urca process losses, which are negligible at the temperatures of interest here (cf. Beaudet, Salpeter, and Silvestro 1972).

Energy production rates for the proton-proton chain and the $\mathrm{CN}$ cycle were calculated using the unscreened expressions of Reeves (1965). The initial abundance of $\mathrm{CN}$ catalyst nuclei was assumed equal to half the total heavy-element abundance. The triplealpha and alpha-carbon reaction rates have been modified from the original expressions of Fowler, Caughlan, and Zimmerman (1967) to reflect new nuclear data on these reactions. Screening corrections were included.

\section{b) The Evolution Program}

The theoretical evolution calculations were performed using a modified version of the University of Texas copy of B. Paczyński's $(1969,1970)$ stellarevolution code. The standard Henyey technique (Henyey et al. 1959; Henyey, Forbes, and Gould 1964) is used to converge each evolutionary interior model. The outer boundary conditions for the interior iterations are interpolated from a table prepared from a grid of model stellar envelopes containing the outer 10 percent of the mass, which were computed prior to the interior calculations. Details can be found in Paczyński (1969, 1970, and references therein). Since essentially all of the input physics required is inter- polated from tables, rather than computed at each step, the program is relatively fast.

\section{c) The Analysis Program}

A computer program written by the author was used to perform pulsation and stability calculations for complete stellar models produced by fitting a model envelope to an interior model selected from an evolutionary sequence. Assuming linear quasi-adiabatic oscillations, the frequency eigenvalue for the fundamental radial pulsation mode was then obtained using the iterative fitting method of Bardeen, Thorne, and Meltzer (1966; cf. Christy 1966, 1967) to determine the radial eigensolutions for successive estimates of the eigenvalue. The derivatives of the eigensolution were matched to better than 0.1 percent at the fitting point $\left(m_{\mathrm{fit}} / M=0.9\right)$. The accuracy of this procedure has been tested using two polytropic models for which the fundamental-mode pulsation constants, $Q_{0} \equiv$ $\Pi_{0}\left(\rho / \rho_{\odot}\right)^{1 / 2}$, where $\Pi_{0}$ is the fundamental period in days and the densities are mean values, are known analytically. Computations for polytropes of indices $n=0$ (uniform density) and $n=3 / 2$ with adiabatic exponent $\gamma=5 / 3$ yielded pulsation constants $Q_{0}=$ 0.1165 and 0.0707 , respectively. Analytic solutions yield corresponding values of $Q_{0}=0.1158$ and 0.0703 (Ledoux and Walraven 1958).

The stability of a mass shell in a given model can be estimated by means of the "one-zero" approximation of Baker (1966), writing the linearized pulsation equation for the shell as

$$
\ddot{r}^{\prime}+\sigma_{0} K A \ddot{r}^{\prime}+\sigma_{0}^{2} B \dot{r}^{\prime}+\sigma_{0}^{3} K D r^{\prime}=0,
$$

where the dot denotes differentiation with respect to time and

$$
\sigma_{0}^{2}=G m r_{0}^{-3}, \quad K=2 L_{0} \rho_{0} / \sigma_{0} m P_{0} \delta .
$$

The coefficients $A, B, D$ are given by

$$
\begin{aligned}
A= & -\left[\alpha\left(l \epsilon_{T}+\kappa_{T}-4\right)+\delta\left(l \epsilon_{p}+\kappa_{p}\right)\right](\alpha C-\delta)^{-1}, \\
B= & {[4 \delta-C(4 \alpha-3)](\alpha C-\delta)^{-1}, } \\
D= & {\left[4 \delta\left(l \epsilon_{p}+\kappa_{p}+1\right)\right.} \\
& \left.+(4 \alpha-3)\left(l \epsilon_{T}+\kappa_{T}-4\right)\right](\alpha C-\delta)^{-1} .
\end{aligned}
$$

A zero subscript denotes the equilibrium value of a variable and we have defined

$$
\begin{gathered}
\left.\left.\alpha \equiv \frac{\partial \ln \rho_{0}}{\partial \ln p_{0}}\right)_{T}, \quad \delta \equiv-\frac{\partial \ln \rho_{0}}{\partial \ln T_{0}}\right)_{p}, \quad C \equiv \frac{\mu \beta}{\delta R} C_{p}, \\
\left.\left.\kappa_{p} \equiv \frac{\partial \ln \kappa_{0}}{\partial \ln p_{0}}\right)_{T}, \quad \kappa_{T} \equiv \frac{\partial \ln \kappa_{0}}{\partial \ln T_{0}}\right)_{p}, \quad l \equiv \frac{M}{2 L_{0}}, \\
\epsilon_{p} \equiv \frac{\partial}{\partial \ln p_{0}} \ln \left(\epsilon_{0}^{\text {nuc }}-\epsilon_{0}{ }^{v}\right)_{T}, \\
\epsilon_{T} \equiv \frac{\partial}{\partial \ln T_{0}} \ln \left(\epsilon_{0}^{\text {nuc }}-\epsilon_{0}{ }^{v}\right)_{p} .
\end{gathered}
$$

Other symbols have their usual meaning. 

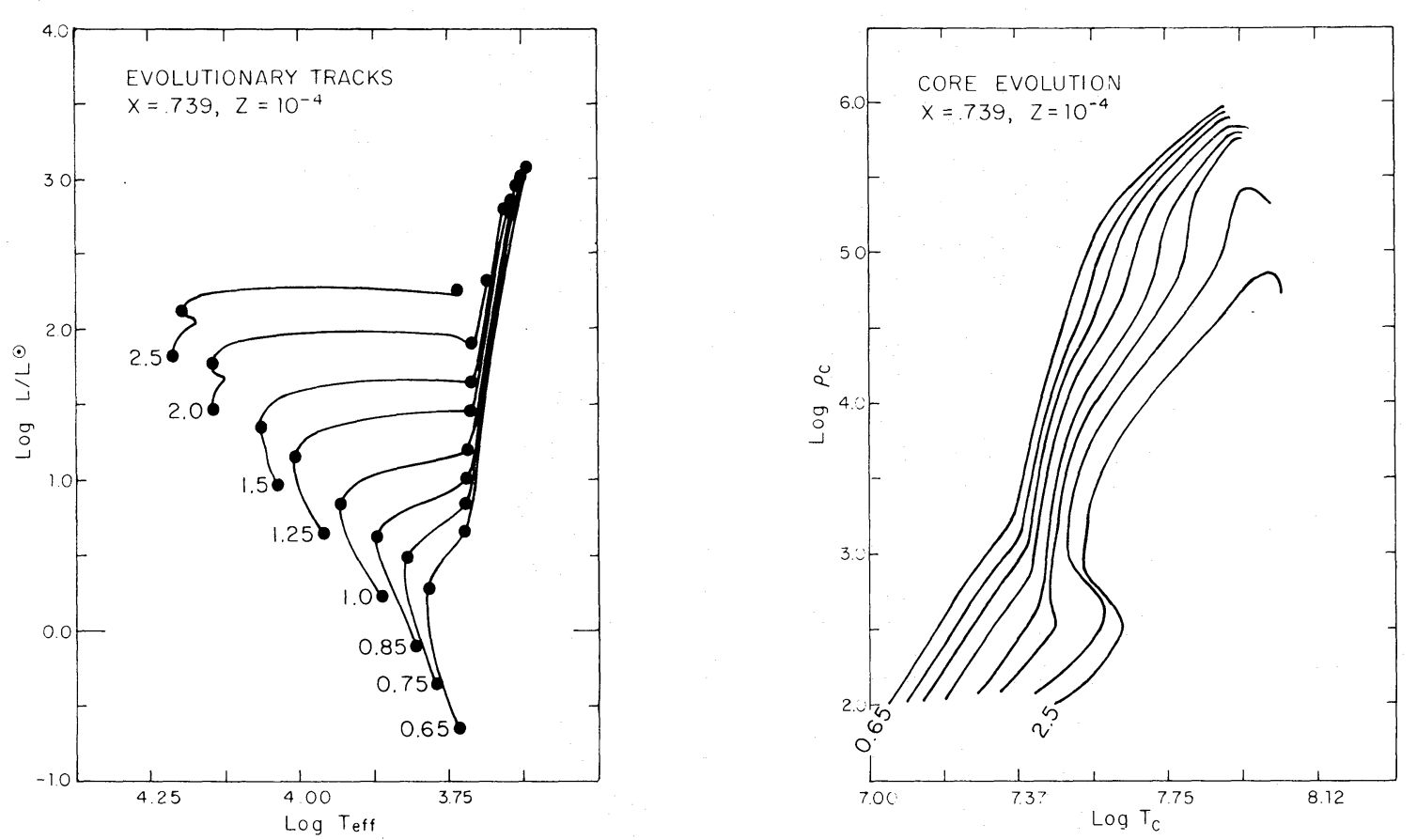

FIG. 1a (left $)$.-Evolutionary tracks for $(X, Z)=\left(0.739,10^{-4}\right)$. Filled circles indicate models at the principal points discussed in the text.

FIG. $1 b$ (right).-Core evolution for $(X, Z)=\left(0.739,10^{-4}\right)$.
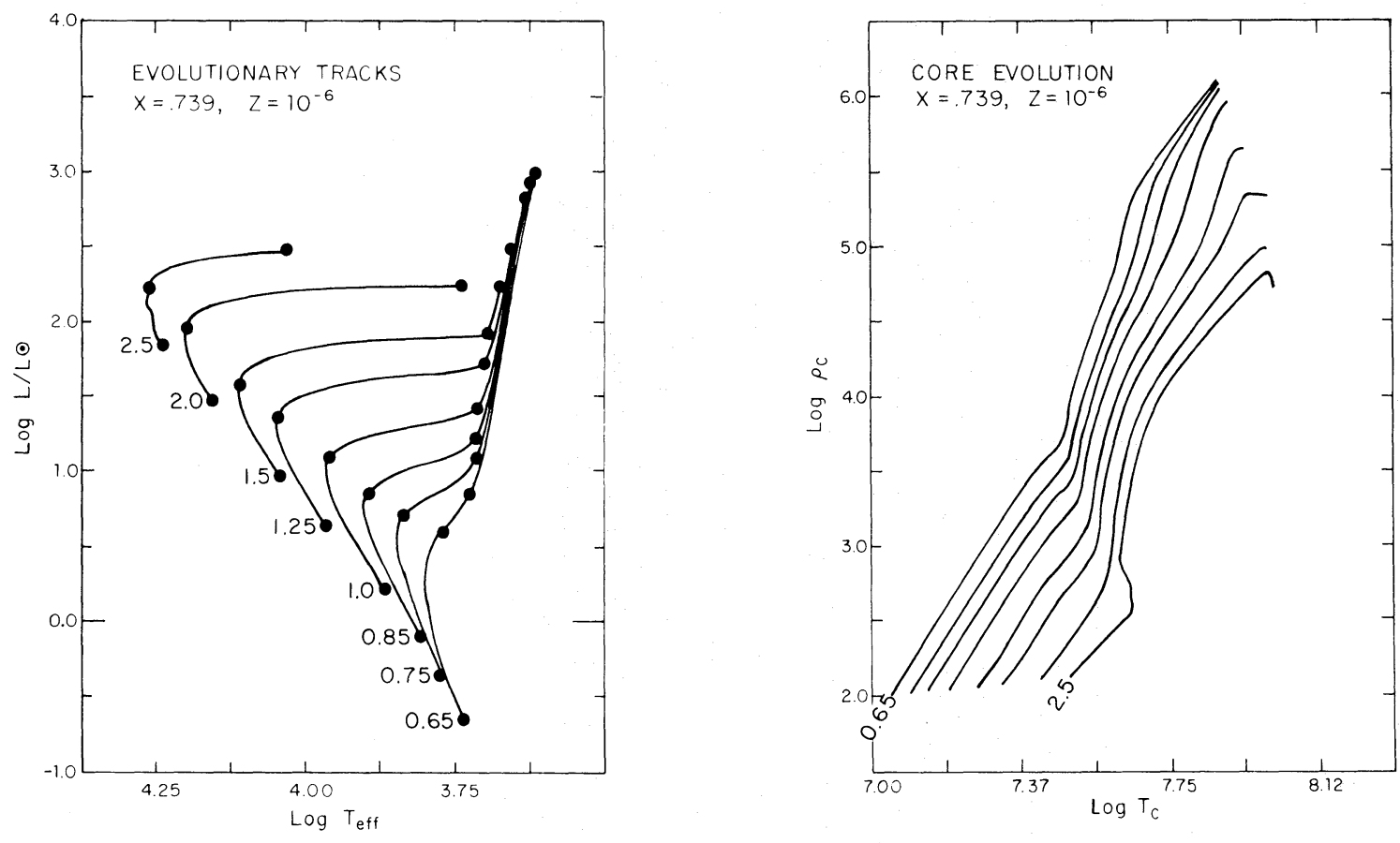

FIG. $2 a$ (left).-Evolutionary tracks for $(X, Z)=\left(0.739,10^{-6}\right)$. Points as in fig. $1 a$.

FIG. $2 b$ (right).-Core evolution for $(X, Z)=\left(0.739,10^{-6}\right)$. 

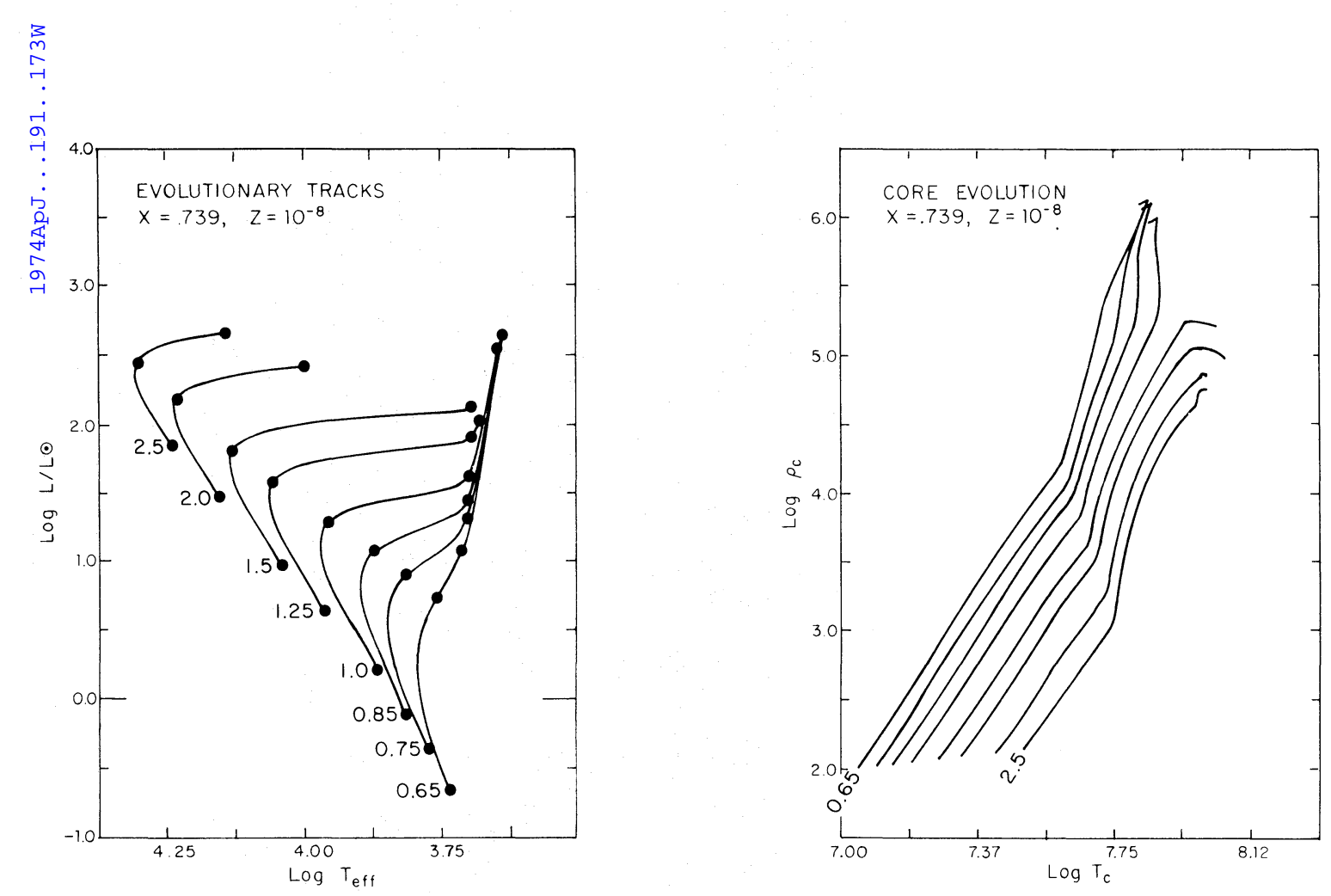

Fig. $3 a$ (left $)$.-Evolutionary tracks for $(X, Z)=\left(0.739,10^{-8}\right)$. Points as in fig. $1 a$.

FIg. $3 b$ (right).-Core evolution for $(X, Z)=\left(0.739,10^{-8}\right)$.
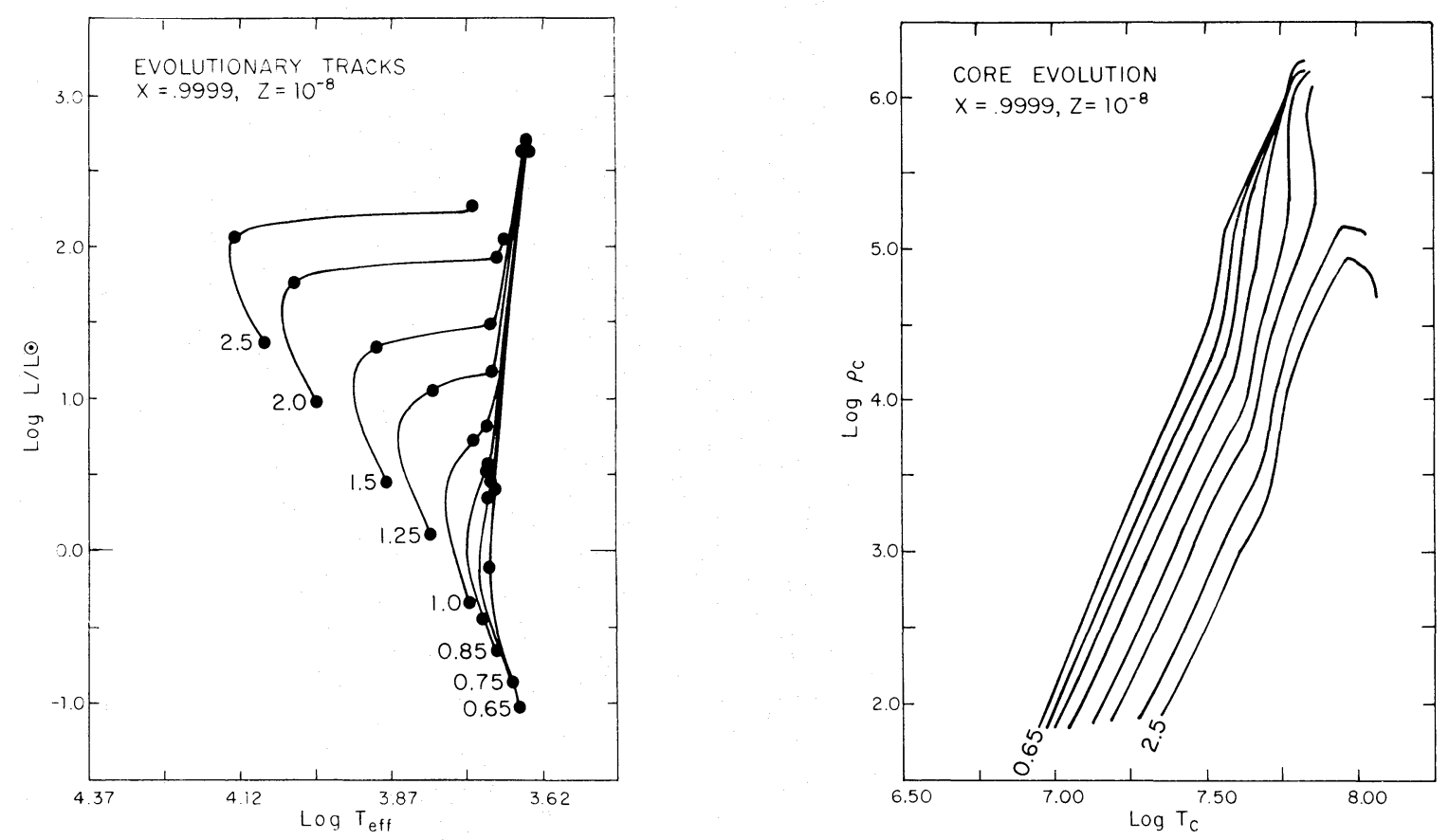

Fig. $4 a$ (left).-Evolutionary tracks for $(X, Z)=\left(0.9999,10^{-8}\right)$. Points as in fig. $1 a$.

Fig. $4 b$ (right).-Core evolution for $(X, Z)=\left(0.9999,10^{-8}\right)$. 
If we now assume that the radial pulsation can be written as

$$
\begin{aligned}
r(m, t) & =r_{0}(m)\left[1+r^{\prime}(m, t)\right] \\
& =r_{0}(m)\left[1+r^{\prime}(m) e^{i \omega t}\right],
\end{aligned}
$$

then the pulsation equation (1) becomes

$$
(i \omega)^{3}+\sigma_{0} K A(i \omega)^{2}+\sigma_{0}^{2} B(i \omega)+\sigma_{0}^{3} K D=0 .
$$

In the general nonadiabatic case, $K \neq 0$, and the requirement of stable oscillations (e.g., that the three roots of eq. [6] have positive imaginary parts) implies that

$\sigma_{0}{ }^{2} B>0, \sigma_{0}^{3} K D>0$, and $\sigma_{0}^{3} K(A B-D)>0$.

The first and second conditions are required for dynamical and secular stability of the mass shell. The remaining condition must be met if the shell is to be pulsationally stable. Using the "one-zone" approximation, therefore, we can estimate the overall stability of a model by means of an appropriate average of the parameters (7) through the model. Zone interactions and variations of the variables within each zone are of course neglected in this estimate. For a typical model, several hundred mass shells were included in the averages. Both mass averaging and radius averaging yielded similar results. None of the models analyzed in this way was found to be secularly unstable. An advantage of this procedure is that it provides one with a picture of which regions of the stellar model produce the instability, if it is present.

\section{THEORETICAL EVOLUTION}

Evolutionary sequences from zero-age main sequence to central helium ignition have been computed for stars of mass $0.65,0.75,0.85,1.00,1.25,1.50,2.00$, $2.50 M_{\odot}$ and compositions $(X, Z)=\left(0.739,10^{-4}\right)$, $\left(0.739,10^{-6}\right),\left(0.739,10^{-8}\right)$, and $\left(0.9999,10^{-8}\right)$. The evolutionary tracks in the (luminosity, effective temperature)- and (central density, central temperature)-planes are presented in figures 1-4. The points indicated along the tracks denote models listed in tables 1-4: the uniform initial main-sequence model $(M S)$, the model in which central exhaustion of hydrogen occurs $\left(X_{c}\right)$, the hydrogen shell-source model in which the envelope convection first reaches below the exterior fitting point $(C E)$, and the model in which the central density reaches its maximum value during ignition of the helium-rich core $\left(\rho_{c}\right)$. Column (1) gives the mass of the stellar model in solar units, and column (13) indicates the evolutionary stage. Columns (2) through (7) list, respectively, the model age in billions of years, the logarithm of the luminosity (solar units), the logarithm of the effective temperature $\left({ }^{\circ} \mathrm{K}\right)$, the radius (solar units), the logarithm of the central density $\left(\mathrm{g} \mathrm{cm}^{-3}\right)$, and the logarithm of the central temperature $\left({ }^{\circ} \mathrm{K}\right)$. Columns $(8)$ through (12) give the mass of the convective core if present, the mass of the hydrogen-exhausted region, and the mass to which

\begin{tabular}{|c|c|c|c|c|c|c|c|c|c|c|c|c|}
\hline Mass & Age & $\log L$ & $\log \mathrm{T}_{\mathrm{e}}$ & $\mathrm{R}$ & $\log \rho_{c}$ & $\log \mathrm{T}_{\mathrm{c}}$ & $\mathrm{M}_{\mathrm{cc}}$ & $\mathrm{M}_{\mathrm{x}}$ & $\mathrm{M}_{\mathrm{ce}}$ & $\pi_{0}$ & $Q_{0}$ & \\
\hline 0.65 & $\begin{array}{l}0.0 \\
20.249 \\
21.107 \\
21.597\end{array}$ & $\begin{array}{l}-.649 \\
0.264 \\
0.649 \\
3.079\end{array}$ & $\begin{array}{l}3.736 \\
3.790 \\
3.727 \\
3.621\end{array}$ & $\begin{array}{l}2.49 \\
66.5\end{array}$ & $\begin{array}{l}2.009 \\
3.573 \\
4.567 \\
5.960\end{array}$ & $\begin{array}{l}7.047 \\
7.387 \\
7.495 \\
7.885\end{array}$ & $\begin{array}{c}.003 \\
-- \\
-- \\
.176\end{array}$ & $\begin{array}{l}-- \\
.010 \\
.142 \\
.476\end{array}$ & $\begin{array}{l}-- \\
-- \\
0.584 \\
0.489\end{array}$ & $\begin{array}{l}0.029 * \\
0.083 \\
0.235 \\
37.75\end{array}$ & $\begin{array}{l}.059 \\
.051 \\
.048 \\
.060\end{array}$ & $\begin{array}{l}\mathrm{MS} \\
\mathrm{X}_{\mathrm{C}} \\
\mathrm{CE} \\
\rho_{\mathrm{C}}\end{array}$ \\
\hline 0.75 & $\begin{array}{l}0.0 \\
11.524 \\
12.497 \\
12.811\end{array}$ & $\begin{array}{l}-.357 \\
0.458 \\
0.835 \\
3.006\end{array}$ & $\begin{array}{l}3.774 \\
3.826 \\
3.726 \\
3.626\end{array}$ & $\begin{array}{l}0.63 \\
1.27 \\
3.09 \\
59.7\end{array}$ & $\begin{array}{l}2.021 \\
3.461 \\
4.587 \\
5.920\end{array}$ & & $\begin{array}{l}.007 \\
-- \\
-- \\
--\end{array}$ & $\begin{array}{l}-- \\
.011 \\
.157 \\
.466\end{array}$ & $\begin{array}{l}-- \\
-- \\
0.673 \\
0.481\end{array}$ & & $\begin{array}{l}.054 \\
.046 \\
.048 \\
.060\end{array}$ & $\begin{array}{l}\mathrm{MS} \\
\mathrm{X}_{\mathrm{C}} \\
\mathrm{CE} \\
\rho_{\mathrm{C}}\end{array}$ \\
\hline 0.85 & $\begin{array}{c}0.0 \\
7.468 \\
7.978 \\
8.194\end{array}$ & $\begin{array}{l}-.104 \\
0.629 \\
0.994 \\
3.014\end{array}$ & $\begin{array}{l}3.808 \\
3.873 \\
3.725 \\
3.627\end{array}$ & $\begin{array}{l}1.24 \\
3.73 \\
59.9\end{array}$ & $\begin{array}{l}2.032 \\
3.372 \\
4.615 \\
5.896\end{array}$ & 10 & $\begin{array}{c}.011 \\
-- \\
-- \\
--\end{array}$ & $\begin{array}{l}-- \\
.011 \\
.168 \\
.456\end{array}$ & $\begin{array}{l}-- \\
-\overline{-} \\
0.764 \\
0.472\end{array}$ & & $\begin{array}{l}.053 \\
.045 \\
.048 \\
.060\end{array}$ & $\begin{array}{l}\mathrm{MS} \\
\mathrm{X}_{\mathrm{C}} \\
\mathrm{CE} \\
\rho_{\mathrm{C}}\end{array}$ \\
\hline 1.00 & $\begin{array}{l}0.0 \\
4.151 \\
4.539 \\
4.674\end{array}$ & $\begin{array}{l}0.217 \\
0.844 \\
1.199 \\
2.947\end{array}$ & $\begin{array}{l}3.865 \\
3.932 \\
3.721 \\
3.636\end{array}$ & $\begin{array}{l}0.80 \\
1.21 \\
4.80 \\
53.3\end{array}$ & $\begin{array}{l}2.047 \\
3.248 \\
4.683 \\
5.832\end{array}$ & 7.924 & $\begin{array}{c}.009 \\
-- \\
-- \\
--\end{array}$ & $\begin{array}{l}-- \\
.011 \\
.1 .91 \\
.439\end{array}$ & $\begin{array}{l}-- \\
-- \\
0.897 \\
0.459\end{array}$ & & $\begin{array}{l}.048 \\
.046 \\
.048 \\
.058\end{array}$ & $\begin{array}{l}\mathrm{MS} \\
\mathrm{X}_{\mathrm{C}} \\
\mathrm{CE} \\
\rho_{\mathrm{C}}\end{array}$ \\
\hline 1.25 & $\begin{array}{c}0.0 \\
1.881 \\
2.159 \\
2.234\end{array}$ & $\begin{array}{l}0.642 \\
1.129 \\
1.455 \\
2.849\end{array}$ & $\begin{array}{l}3.962 \\
4.008 \\
3.718 \\
3.648\end{array}$ & $\begin{array}{l}0.83 \\
1.18 \\
6.56 \\
45.1\end{array}$ & $\begin{array}{l}2.070 \\
3.079 \\
4.783 \\
5.747\end{array}$ & $\begin{array}{l}7.266 \\
7.446 \\
7.708 \\
7.923\end{array}$ & $\begin{array}{l}.027 \\
-- \\
-- \\
--\end{array}$ & $\begin{array}{l}-- \\
.012 \\
.222 \\
.417\end{array}$ & $\begin{array}{l}-- \\
-- \\
1.121 \\
0.447\end{array}$ & $\begin{array}{l}0.032 \\
0.058 \\
0.726 \\
15.10\end{array}$ & $\begin{array}{l}.047 \\
.049 \\
.048 \\
.057\end{array}$ & $\begin{array}{l}\mathrm{MS} \\
\mathrm{X}_{\mathrm{C}} \\
\mathrm{CE} \\
\rho_{\mathrm{C}}\end{array}$ \\
\hline 1.50 & $\begin{array}{c}0.0 \\
1.004 \\
1.209 \\
1.257\end{array}$ & $\begin{array}{l}0.974 \\
1.363 \\
1.644 \\
2.770\end{array}$ & $\begin{array}{l}4.038 \\
4.065 \\
3.716 \\
3.657\end{array}$ & $\begin{array}{l}1.19 \\
8.22 \\
39.4\end{array}$ & $\begin{array}{l}2.084 \\
2.974 \\
4.871 \\
5.687\end{array}$ & $\begin{array}{l}7.327 \\
7.462 \\
7.779 \\
7.936\end{array}$ & $\begin{array}{l}.078 \\
-- \\
-- \\
--\end{array}$ & $\begin{array}{l}-- \\
.017 \\
.249 \\
.403\end{array}$ & $\begin{array}{l}-- \\
-- \\
1.345 \\
0.442\end{array}$ & $\begin{array}{l}0.031 \\
0.048 \\
0.929 \\
11.48\end{array}$ & $\begin{array}{l}.048 \\
.045 \\
.048 \\
.058\end{array}$ & $\begin{array}{l}\mathrm{MS} \\
\mathrm{X}_{\mathrm{C}} \\
\mathrm{CE} \\
\rho_{\mathrm{C}}\end{array}$ \\
\hline 2.00 & $\begin{array}{c}0.0 \\
0.402 \\
0.485 \\
0.502\end{array}$ & $\begin{array}{l}1.464 \\
1.782 \\
1.905 \\
2.314\end{array}$ & $\begin{array}{l}4.145 \\
4.145 \\
3.715 \\
3.685\end{array}$ & $\begin{array}{l}1.33 \\
11.2 \\
20.5\end{array}$ & $\begin{array}{l}2.072 \\
3.062 \\
5.029 \\
5.426\end{array}$ & $\begin{array}{l}7.414 \\
7.497 \\
7.893 \\
7.959\end{array}$ & $\begin{array}{l}.243 \\
-- \\
-- \\
.016\end{array}$ & $\begin{array}{l}-- \\
.041 \\
.299 \\
.350\end{array}$ & $\begin{array}{c}-. \\
-- \\
1.796 \\
0.533\end{array}$ & $\begin{array}{l}0.031 \\
0.050 \\
1.282 \\
3.618^{*}\end{array}$ & $\begin{array}{l}.049 \\
.046 \\
.049 \\
.057\end{array}$ & $\begin{array}{l}\mathrm{MS} \\
\mathrm{X}_{\mathrm{C}} \\
\mathrm{CE} \\
\rho_{\mathrm{C}}\end{array}$ \\
\hline 2.50 & $\begin{array}{c}0.0 \\
0.234 \\
0.261\end{array}$ & $\begin{array}{l}1.819 \\
2.141 \\
2.227\end{array}$ & $\begin{array}{l}4.212 \\
4.193 \\
3.754\end{array}$ & $\begin{array}{l}1.03 \\
1.62 \\
13.5\end{array}$ & $\begin{array}{l}2.004 \\
2.967 \\
4.854\end{array}$ & $\begin{array}{l}7.460 \\
7.537 \\
8.000\end{array}$ & $\begin{array}{l}.477 \\
-0 \\
.017\end{array}$ & $\begin{array}{l}-- \\
.045 \\
.345\end{array}$ & -- & $\begin{array}{l}0.032 \\
0.060 \\
1.991 *\end{array}$ & $\begin{array}{l}.049 \\
.046 \\
.054\end{array}$ & $\begin{array}{l}\mathrm{MS} \\
\mathrm{X}_{\mathrm{C}} \\
\rho_{\mathrm{c}}\end{array}$ \\
\hline
\end{tabular}

TABLE 1

Principal Points: $X=0.739, Z=10^{-4}$ 
TABLE 2

Principal Points: $X=0.739, Z=10^{-6}$

\begin{tabular}{|c|c|c|c|c|c|c|c|c|c|c|c|c|}
\hline Mass & Age & $\log L$ & $\log \mathrm{T}_{\mathrm{e}}$ & $\mathrm{R}$ & $\log \rho_{c}$ & $\log \mathrm{T}_{\mathrm{c}}$ & $\mathrm{M}_{\mathrm{cc}}$ & $\mathrm{M}_{\mathrm{x}}$ & $\mathrm{M}_{\mathrm{ce}}$ & II & $Q_{0}$ & \\
\hline 0.65 & $\begin{array}{c}0.0 \\
20.898 \\
21.389 \\
21.762\end{array}$ & $\begin{array}{l}-.647 \\
0.510 \\
0.906 \\
2.982\end{array}$ & $\begin{array}{l}3.736 \\
3.789 \\
3.716 \\
3.617\end{array}$ & $\begin{array}{l}0.54 \\
1.59 \\
3.51 \\
60.4\end{array}$ & $\begin{array}{l}2.009 \\
3.978 \\
4.869 \\
6.106\end{array}$ & $\begin{array}{l}7.048 \\
7.503 \\
7.615 \\
7.868\end{array}$ & $\begin{array}{c}.003 \\
-- \\
-- \\
--\end{array}$ & $\begin{array}{l}-- \\
.013 \\
.175 \\
.515\end{array}$ & $\begin{array}{c}-- \\
-- \\
0.582 \\
0.538\end{array}$ & $\begin{array}{l}0.029 * \\
0.124 * \\
0.390 \\
31.58\end{array}$ & $\begin{array}{l}.060 \\
.050 \\
.048 \\
.058\end{array}$ & $\begin{array}{l}\mathrm{MS} \\
\mathrm{X}_{\mathrm{C}} \\
\mathrm{CE} \\
\rho_{\mathrm{C}}\end{array}$ \\
\hline 0.75 & $\begin{array}{c}0.0 \\
12.363 \\
12.709 \\
12.956\end{array}$ & $\begin{array}{l}-.354 \\
0.699 \\
1.071 \\
2.965\end{array}$ & $\begin{array}{l}3.775 \\
3.841 \\
3.716 \\
3.618\end{array}$ & $\begin{array}{l}0.63 \\
1.56 \\
4.25 \\
58.9\end{array}$ & $\begin{array}{l}2.021 \\
3.872 \\
4.838 \\
6.087\end{array}$ & $\begin{array}{l}7.095 \\
7.519 \\
7.649 \\
7.871\end{array}$ & $\begin{array}{l}.007 \\
-- \\
-- \\
--\end{array}$ & $\begin{array}{l}-- \\
.013 \\
.183 \\
.510\end{array}$ & $\begin{array}{c}-- \\
-- \\
0.672 \\
0.536\end{array}$ & $\begin{array}{l}0.033 \\
0.102 \\
0.481 \\
28.72\end{array}$ & $\begin{array}{l}.052 \\
.045 \\
.048 \\
.058\end{array}$ & $\begin{array}{l}\mathrm{MS} \\
\mathrm{X}_{\mathrm{C}} \\
\mathrm{CE} \\
\rho_{\mathrm{C}}\end{array}$ \\
\hline 0.85 & $\begin{array}{c}0.0 \\
7.891 \\
8.163 \\
8.322\end{array}$ & $\begin{array}{l}-.101 \\
0.864 \\
1.242 \\
2.933\end{array}$ & $\begin{array}{l}3.810 \\
3.896 \\
3.712 \\
3.622\end{array}$ & $\begin{array}{l}0.72 \\
1.46 \\
5.26 \\
56.0\end{array}$ & $\begin{array}{l}2.032 \\
3.777 \\
4.863 \\
6.053\end{array}$ & $\begin{array}{l}7.138 \\
7.532 \\
7.685 \\
7.877\end{array}$ & $\begin{array}{l}.011 \\
-- \\
-- \\
--\end{array}$ & $\begin{array}{l}-- \\
.013 \\
.204 \\
.501\end{array}$ & $\begin{array}{l}-- \\
-- \\
0.760 \\
0.530\end{array}$ & $\begin{array}{l}0.035 \\
0.085 \\
0.627 \\
25.32\end{array}$ & $\begin{array}{l}.052 \\
.045 \\
.048 \\
.058\end{array}$ & $\begin{array}{l}\mathrm{MS} \\
\mathrm{X}_{\mathrm{C}} \\
\mathrm{CE} \\
\rho_{\mathrm{C}}\end{array}$ \\
\hline 1.00 & $\begin{array}{c}0.0 \\
4.487 \\
4.683 \\
4.776\end{array}$ & $\begin{array}{l}0.219 \\
1.078 \\
1.439 \\
2.834\end{array}$ & $\begin{array}{l}3.869 \\
3.965 \\
3.710 \\
3.631\end{array}$ & $\begin{array}{l}0.79 \\
1.36 \\
6.67 \\
47.9\end{array}$ & $\begin{array}{l}2.047 \\
3.654 \\
4.864 \\
5.962\end{array}$ & $\begin{array}{l}7.192 \\
7.548 \\
7.731 \\
7.896\end{array}$ & $\begin{array}{l}.008 \\
-- \\
-- \\
--\end{array}$ & $\begin{array}{l}-- \\
.013 \\
.218 \\
.476\end{array}$ & $\begin{array}{l}-- \\
-- \\
0.899 \\
0.517\end{array}$ & $\begin{array}{l}0.034 \\
0.070 \\
0.819 \\
18.37\end{array}$ & $\begin{array}{l}.048 \\
.044 \\
.048 \\
.057\end{array}$ & $\begin{array}{l}\mathrm{MS} \\
\mathrm{X}_{\mathrm{C}} \\
\mathrm{CE} \\
\rho_{\mathrm{C}}\end{array}$ \\
\hline 1.25 & $\begin{array}{c}0.0 \\
2.124 \\
2.254 \\
2.292\end{array}$ & $\begin{array}{l}0.644 \\
1.368 \\
1.712 \\
2.498\end{array}$ & $\begin{array}{l}3.966 \\
4.049 \\
3.706 \\
3.655\end{array}$ & $\begin{array}{l}0.82 \\
1.29 \\
9.31 \\
29.1\end{array}$ & $\begin{array}{l}2.071 \\
3.499 \\
4.912 \\
5.656\end{array}$ & $\begin{array}{l}7.266 \\
7.571 \\
7.807 \\
7.951\end{array}$ & $\begin{array}{l}.026 \\
-- \\
-- \\
.003\end{array}$ & $\begin{array}{l}-- \\
.014 \\
.252 \\
.399\end{array}$ & $\begin{array}{l}-- \\
-- \\
1.122 \\
0.508\end{array}$ & $\begin{array}{l}0.032 \\
0.058 \\
1.209 \\
7.619\end{array}$ & $\begin{array}{l}.047 \\
.044 \\
.048 \\
.055\end{array}$ & $\begin{array}{l}\mathrm{MS} \\
\mathrm{X}_{\mathrm{C}} \\
\mathrm{CE} \\
\rho_{\mathrm{C}}\end{array}$ \\
\hline 1.50 & $\begin{array}{c}0.0 \\
1.177 \\
1.275 \\
1.288\end{array}$ & $\begin{array}{l}0.976 \\
1.595 \\
1.920 \\
2.243\end{array}$ & $\begin{array}{l}4.041 \\
4.112 \\
3.701 \\
3.673\end{array}$ & $\begin{array}{l}0.85 \\
1.25 \\
12.1 \\
19.9\end{array}$ & $\begin{array}{l}2.087 \\
3.367 \\
4.974 \\
5.355\end{array}$ & $\begin{array}{l}7.328 \\
7.587 \\
7.876 \\
7.958\end{array}$ & $\begin{array}{l}.066 \\
-- \\
-- \\
.003\end{array}$ & $\begin{array}{l}-- \\
.015 \\
.287 \\
.348\end{array}$ & $\begin{array}{l}-- \\
-- \\
1.347 \\
0.690\end{array}$ & $\begin{array}{l}0.031 \\
0.051 \\
1.640 \\
3.893 *\end{array}$ & $\begin{array}{l}.048 \\
.045 \\
.048 \\
.054\end{array}$ & $\begin{array}{l}\mathrm{MS} \\
\mathrm{X}_{\mathrm{C}} \\
\mathrm{CE} \\
\rho_{\mathrm{C}}\end{array}$ \\
\hline 2.00 & $\begin{array}{c}0.0 \\
0.478 \\
0.542\end{array}$ & $\begin{array}{l}1.476 \\
1.941 \\
2.243\end{array}$ & $\begin{array}{l}4.156 \\
4.201 \\
3.748\end{array}$ & $\begin{array}{l}0.89 \\
1.24 \\
14.1\end{array}$ & $\begin{array}{l}2.117 \\
3.163 \\
4.997\end{array}$ & $\begin{array}{l}7.424 \\
7.613 \\
7.971\end{array}$ & $\begin{array}{l}.052 \\
-- \\
--\end{array}$ & $\begin{array}{l}-\overline{-} \\
.017 \\
.338\end{array}$ & $\begin{array}{l}-- \\
-- \\
--\end{array}$ & $\begin{array}{l}0.029 \\
0.045 \\
2.182 *\end{array}$ & $\begin{array}{l}.049 \\
.046 \\
.056\end{array}$ & $\begin{array}{l}\mathrm{MS} \\
\mathrm{X}_{\mathrm{c}} \\
\rho_{\mathrm{c}}\end{array}$ \\
\hline 2.50 & $\begin{array}{c}0.0 \\
0.242 \\
0.283\end{array}$ & $\begin{array}{l}1.846 \\
2.217 \\
2.474\end{array}$ & $\begin{array}{l}4.239 \\
4.262 \\
4.033\end{array}$ & $\begin{array}{l}0.93 \\
1.29 \\
4.96\end{array}$ & $\begin{array}{l}2.136 \\
3.099 \\
4.829\end{array}$ & $\begin{array}{l}7.496 \\
7.633 \\
8.004\end{array}$ & $\begin{array}{l}.179 \\
-0 \\
.006\end{array}$ & $\begin{array}{l}-.- \\
.036 \\
.361\end{array}$ & $\begin{array}{l}-- \\
-- \\
--\end{array}$ & $\begin{array}{l}0.028 \\
0.042 \\
0.315 *\end{array}$ & $\begin{array}{l}.049 \\
.046 \\
.045\end{array}$ & $\begin{array}{l}\mathrm{MS} \\
\mathrm{X}_{\mathrm{c}} \\
\rho_{\mathrm{c}}\end{array}$ \\
\hline
\end{tabular}

TABLE 3

Principal PoInTs: $X=0.739, Z=10^{-8}$

\begin{tabular}{|c|c|c|c|c|c|c|c|c|c|c|c|c|}
\hline Mass & Age & $\log \mathrm{L}$ & $\log \mathrm{T}_{\mathrm{e}}$ & $\mathrm{R}$ & $\log \rho_{c}$ & $\log \mathrm{T}_{\mathrm{c}}$ & $M_{c c}$ & $M_{x}$ & $\mathrm{M}_{\mathrm{ce}}$ & $\Gamma_{0}$ & $Q_{0}$ & \\
\hline 0.65 & $\begin{array}{c}0.0 \\
21.456 \\
21.752 \\
22.047\end{array}$ & $\begin{array}{l}-.647 \\
0.750 \\
1.097 \\
2.663\end{array}$ & $\begin{array}{l}3.737 \\
3.762 \\
3.717 \\
3.638\end{array}$ & $\begin{array}{l}0.53 \\
2.38 \\
4.36 \\
38.1\end{array}$ & $\begin{array}{l}2.009 \\
4.412 \\
5.074 \\
6.131\end{array}$ & $\begin{array}{l}7.048 \\
7.624 \\
7.699 \\
7.846\end{array}$ & $\begin{array}{l}.003 \\
-- \\
-- \\
--\end{array}$ & $\begin{array}{l}-- \\
.019 \\
.186 \\
.519\end{array}$ & $\begin{array}{c}-- \\
-- \\
0.584 \\
0.574\end{array}$ & $\begin{array}{l}0.028 * \\
0.244 \\
0.537 \\
14.94\end{array}$ & $\begin{array}{l}.057 \\
.054 \\
.048 \\
.055\end{array}$ & $\begin{array}{l}\mathrm{MS} \\
\mathrm{X}_{\mathrm{C}} \\
\mathrm{CE} \\
\rho_{\mathrm{C}}\end{array}$ \\
\hline 0.75 & $\begin{array}{c}0.0 \\
12.734 \\
12.949 \\
13.143\end{array}$ & $\begin{array}{l}-.353 \\
0.926 \\
1.279 \\
2.656\end{array}$ & $\begin{array}{l}3.775 \\
3.812 \\
3.708 \\
3.638\end{array}$ & $\begin{array}{l}0.63 \\
2.31 \\
5.60 \\
37.8\end{array}$ & $\begin{array}{l}2.021 \\
4.305 \\
5.095 \\
6.121\end{array}$ & $\begin{array}{l}7.095 \\
7.644 \\
7.748 \\
7.848\end{array}$ & $\begin{array}{l}.007 \\
-- \\
-- \\
--\end{array}$ & $\begin{array}{l}-- \\
.017 \\
.206 \\
.516\end{array}$ & $\begin{array}{l}-- \\
-- \\
0.673 \\
0.582\end{array}$ & $\begin{array}{l}0.033 \\
0.187 \\
0.730 \\
13.80\end{array}$ & $\begin{array}{l}.058 \\
.047 \\
.048 \\
.055\end{array}$ & $\begin{array}{l}M S \\
X_{C} \\
C E \\
\rho_{C}\end{array}$ \\
\hline 0.85 & $\begin{array}{c}0.0 \\
8.169 \\
8.323 \\
8.460\end{array}$ & $\begin{array}{l}-.100 \\
1.083 \\
1.416 \\
2.639\end{array}$ & $\begin{array}{l}3.817 \\
3.876 \\
3.707 \\
3.640\end{array}$ & $\begin{array}{l}0.69 \\
2.06 \\
6.58 \\
36.6\end{array}$ & $\begin{array}{l}2.033 \\
4.210 \\
5.076 \\
6.100\end{array}$ & $\begin{array}{l}7.138 \\
7.659 \\
7.782 \\
7.852\end{array}$ & $\begin{array}{c}.011 \\
-- \\
-- \\
--\end{array}$ & $\begin{array}{l}-- \\
.017 \\
.223 \\
.510\end{array}$ & $\begin{array}{l}-- \\
-- \\
0.764 \\
0.584\end{array}$ & $\begin{array}{l}0.027 \\
0.145 \\
0.871 \\
11.91\end{array}$ & $\begin{array}{l}.044 \\
.045 \\
.048 \\
.055\end{array}$ & $\begin{array}{l}\mathrm{MS} \\
\mathrm{X}_{\mathrm{C}} \\
\mathrm{CE} \\
\rho_{\mathrm{C}}\end{array}$ \\
\hline 1.00 & $\begin{array}{c}0.0 \\
4.696 \\
4.801 \\
4.877\end{array}$ & $\begin{array}{l}0.219 \\
1.293 \\
1.619 \\
2.555\end{array}$ & $\begin{array}{l}3.870 \\
3.958 \\
3.705 \\
3.647\end{array}$ & $\begin{array}{l}0.78 \\
1.80 \\
8.41 \\
32.2\end{array}$ & $\begin{array}{l}2.047 \\
4.092 \\
5.067 \\
6.011\end{array}$ & $\begin{array}{l}7.193 \\
7.678 \\
7.835 \\
7.871\end{array}$ & $\begin{array}{l}.009 \\
-- \\
-- \\
--\end{array}$ & $\begin{array}{l}-- \\
.017 \\
.245 \\
.485\end{array}$ & $\begin{array}{c}-- \\
-- \\
0.895 \\
0.586\end{array}$ & $\begin{array}{l}0.028 \\
0.110 \\
1.157 \\
9.466\end{array}$ & $\begin{array}{l}.040 \\
.045 \\
.048 \\
.054\end{array}$ & $\begin{array}{l}M S \\
X_{C} \\
C E \\
\rho_{C}\end{array}$ \\
\hline 1.25 & $\begin{array}{c}0.0 \\
2.265 \\
2.332 \\
2.338\end{array}$ & $\begin{array}{l}0.644 \\
1.583 \\
1.908 \\
2.022\end{array}$ & $\begin{array}{l}3.965 \\
4.059 \\
3.697 \\
3.683\end{array}$ & $\begin{array}{l}0.82 \\
1.58 \\
12.2 \\
14.8\end{array}$ & $\begin{array}{l}2.071 \\
3.940 \\
5.084 \\
5.234\end{array}$ & $\begin{array}{l}7.266 \\
7.704 \\
7.908 \\
8.003\end{array}$ & $\begin{array}{c}.026 \\
-- \\
-- \\
--\end{array}$ & $\begin{array}{l}-- \\
.016 \\
.284 \\
.320\end{array}$ & $\begin{array}{c}-- \\
-- \\
1.120 \\
0.914\end{array}$ & $\begin{array}{l}0.032 \\
0.080 \\
1.814 \\
7.619\end{array}$ & $\begin{array}{r}.048 \\
.045 \\
.048 \\
.055\end{array}$ & $\begin{array}{l}\mathrm{MS} \\
\mathrm{X}_{\mathrm{C}} \\
\mathrm{CE} \\
\rho_{\mathrm{C}}\end{array}$ \\
\hline 1.50 & $\begin{array}{c}0.0 \\
1.275 \\
1.323\end{array}$ & $\begin{array}{l}0.976 \\
1.816 \\
2.116\end{array}$ & $\begin{array}{l}4.041 \\
4.132 \\
3.705\end{array}$ & $\begin{array}{l}0.85 \\
1.48 \\
14.9\end{array}$ & $\begin{array}{l}2.087 \\
3.827 \\
5.058\end{array}$ & $\begin{array}{l}7.328 \\
7.723 \\
7.973\end{array}$ & $\begin{array}{l}.066 \\
-- \\
--\end{array}$ & $\begin{array}{l}-- \\
.017 \\
.309\end{array}$ & $\begin{array}{l}-- \\
--\end{array}$ & $\begin{array}{l}0.031 \\
0.065 \\
3.893^{*}\end{array}$ & $\begin{array}{l}.048 \\
.045 \\
.054\end{array}$ & $\begin{array}{l}\mathrm{MS} \\
\mathrm{X}_{\mathrm{C}} \\
\rho_{\mathrm{C}}\end{array}$ \\
\hline 2.00 & $\begin{array}{c}0.0 \\
0.545 \\
0.573\end{array}$ & $\begin{array}{l}1.476 \\
2.171 \\
2.420\end{array}$ & $\begin{array}{l}4.156 \\
4.235 \\
4.002\end{array}$ & $\begin{array}{l}0.89 \\
1.38 \\
5.38\end{array}$ & $\begin{array}{l}2.118 \\
3.628 \\
4.863\end{array}$ & $\begin{array}{l}7.424 \\
7.753 \\
7.985\end{array}$ & $\begin{array}{l}.044 \\
-- \\
--\end{array}$ & $\begin{array}{l}-- \\
.020 \\
.326\end{array}$ & $\begin{array}{l}-- \\
-- \\
--\end{array}$ & $\begin{array}{l}0.032 \\
0.052 \\
2.182 \star\end{array}$ & $\begin{array}{l}.053 \\
.045 \\
.056\end{array}$ & $\begin{array}{l}\mathrm{MS} \\
\mathrm{X}_{\mathrm{C}} \\
\rho_{\mathrm{C}}\end{array}$ \\
\hline 2.50 & $\begin{array}{c}0.0 \\
0.289 \\
0.309\end{array}$ & $\begin{array}{l}1.847 \\
2.434 \\
2.656\end{array}$ & $\begin{array}{l}4.240 \\
4.305 \\
4.146\end{array}$ & $\begin{array}{l}0.93 \\
1.35 \\
3.64\end{array}$ & $\begin{array}{l}2.142 \\
3.485 \\
4.752\end{array}$ & $\begin{array}{l}7.498 \\
7.774 \\
8.002\end{array}$ & $\begin{array}{l}.147 \\
-- \\
.002\end{array}$ & $\begin{array}{l}-- \\
.021 \\
.357\end{array}$ & $\begin{array}{l}-- \\
-- \\
--\end{array}$ & $\begin{array}{l}0.028 \\
0.046 \\
0.315^{*}\end{array}$ & $\begin{array}{l}.049 \\
.046 \\
.045\end{array}$ & $\begin{array}{l}M S \\
X_{C} \\
\rho_{c}\end{array}$ \\
\hline
\end{tabular}


TABLE 4

Principal PoInts: $X=0.9999, Z=10^{-8}$

\begin{tabular}{|c|c|c|c|c|c|c|c|c|c|c|c|c|}
\hline Mass & Age & $\log \mathrm{L}$ & $\log \mathrm{T}_{\mathrm{e}}$ & $\mathrm{R}$ & $\log \rho_{C}$ & $\log \mathrm{T}_{\mathrm{c}}$ & $\mathrm{M}_{\mathrm{cc}}$ & $M_{x}$ & $\mathrm{M}_{\mathrm{ce}}$ & $\pi_{0}$ & $Q_{0}$ & \\
\hline \multirow[t]{4}{*}{0.65} & 0.0 & -1.017 & 3.663 & 0.49 & 1.840 & 6.948 & .022 & -- & 0.394 & $0.026 *$ & .071 & MS \\
\hline & 69.515 & -.150 & 3.713 & 1.06 & 3.862 & 7.379 & -- & -- & 0.584 & $0.067 *$ & .050 & $\mathrm{CE}$ \\
\hline & 73.169 & 0.421 & 3.703 & 2.13 & 4.891 & 7.551 & -- & .041 & 0.465 & 0.199 & .051 & $x_{c}$ \\
\hline & 74.977 & 2.596 & 3.650 & 33.3 & 6.279 & 7.815 & -- & .559 & 0.585 & 12.10 & .052 & $\rho_{\mathrm{c}}$ \\
\hline \multirow[t]{4}{*}{0.75} & 0.0 & -.852 & 3.674 & 0.56 & 1.843 & 6.971 & .011 & -- & 0.622 & $0.028 *$ & .066 & MS \\
\hline & 48.992 & 0.266 & 3.716 & 1.68 & 4.326 & 7.525 & -- & -- & 0.673 & $0.124 *$ & .049 & $\mathrm{CE}$ \\
\hline & 49.753 & 0.459 & 3.708 & 2.18 & 4.723 & 7.574 & -- & .028 & 0.597 & 0.189 & .051 & $\mathrm{X}$ \\
\hline & 51.361 & 2.492 & 3.652 & 29.3 & 6.206 & 7.821 & -- & .537 & 0.588 & 9.319 & .052 & $\rho_{c}^{c}$ \\
\hline \multirow[t]{4}{*}{0.85} & 0.0 & -.648 & 3.670 & 0.63 & 1.850 & 7.002 & .003 & -- & -- & $0.031 *$ & .060 & MS \\
\hline & 32.763 & 0.534 & 3.717 & 2.28 & 4.520 & 7.593 & -- & -- & 0.763 & $0.184 *$ & .049 & $\mathrm{CE}$ \\
\hline & 32.839 & 0.560 & 3.715 & 2.37 & 4.585 & 7.598 & -- & .022 & 0.749 & 0.196 & .049 & $x_{c}$ \\
\hline & 34.096 & 2.666 & 3.646 & 36.9 & 6.291 & 7.828 & -- & .567 & 0.609 & 12.30 & .053 & $\rho_{\mathrm{C}}$ \\
\hline \multirow[t]{4}{*}{1.00} & 0.0 & -.334 & 3.746 & 0.73 & 1.861 & 7.051 & .019 & -- & -- & 0.037 & .059 & MS \\
\hline & 18.468 & 0.762 & 3.732 & 2.76 & 4.415 & 7.624 & -- & .017 & -- & $0.237 *$ & .052 & $\mathrm{x}_{\mathrm{c}}$ \\
\hline & 18.616 & 0.841 & 3.715 & 3.27 & 4.702 & 7.650 & -- & .076 & 0.894 & 0.289 & .049 & $\mathrm{CE}$ \\
\hline & 19.279 & 2.467 & 3.655 & 28.0 & 6.174 & 7.826 & -- & .527 & 0.591 & 7.604 & .052 & $\rho_{c}$ \\
\hline \multirow[t]{4}{*}{1.25} & 0.0 & 0.110 & 3.811 & 0.91 & 1.875 & 7.124 & .048 & -- & -- & 0.040 & .051 & MS \\
\hline & 8.514 & 1.093 & 3.786 & 3.16 & 4.226 & 7.656 & -- & .016 & -- & $0.239 *$ & .048 & $\mathrm{x}_{\mathrm{c}}$ \\
\hline & 8.650 & 1.213 & 3.713 & 5.06 & 4.817 & 7.729 & -- & .148 & 1.122 & $0.489 *$ & .048 & $\mathrm{CE}$ \\
\hline & 8.916 & 2.713 & 3.650 & 38.2 & 6.273 & 7.825 & -- & .561 & 0.613 & 11.11 & .054 & $\rho_{c}$ \\
\hline \multirow[t]{4}{*}{1.50} & 0.0 & 0.459 & 3.883 & 0.97 & 1.886 & 7.184 & .060 & -- & -- & 0.033 & .041 & MS \\
\hline & 4.679 & 1.369 & 3.888 & 2.71 & 4.098 & 7.681 & -- & .017 & -- & $0.164 *$ & .045 & $x_{c}$ \\
\hline & 4.776 & 1.508 & 3.707 & 7.32 & 4.903 & 7.801 & - & .200 & 1.335 & 0.783 & .048 & $\mathrm{CE}$ \\
\hline & 4.889 & 2.639 & 3.656 & 34.0 & 6.160 & 7.845 & -- & .527 & 0.600 & 8.528 & .053 & $\rho_{c}$ \\
\hline \multirow[t]{4}{*}{2.00} & 0.0 & 0.986 & 3.999 & 1.05 & 1.908 & 7.277 & .084 & -- & - & 0.037 & .049 & MS \\
\hline & 1.918 & 1.786 & 4.030 & 2.28 & 3.885 & 7.715 & -- & .017 & -- & 0.105 & .043 & $\mathrm{x}_{\mathrm{c}}$ \\
\hline & 1.972 & 1.933 & 3.699 & 12.4 & 4.985 & 7.909 & -- & .271 & 1.791 & 1.514 & .049 & $\mathrm{CE}$ \\
\hline & 1.977 & 2.056 & 3.687 & 15.1 & 5.161 & 7.979 & .007 & .313 & 1.287 & $2.182 *$ & .053 & $\rho_{c}$ \\
\hline \multirow[t]{3}{*}{2.50} & 0.0 & 1.375 & 4.086 & 1.10 & 1.923 & 7.349 & .181 & -- & -- & 0.036 & .050 & MS \\
\hline & 0.998 & 2.091 & 4.124 & 2.10 & 3.719 & 7.740 & -- & .018 & -- & 0.087 & .046 & $\mathrm{x}_{\mathrm{c}}$ \\
\hline & 1.033 & 2.232 & 3.748 & 14.0 & 4.945 & 7.987 & .002 & .309 & -- & $1.781^{*}$ & .052 & $\rho_{c}$ \\
\hline
\end{tabular}

envelope convection penetrates, all in solar masses, plus the fundamental-mode pulsation period and pulsation constant in days. Pulsational instability is indicated by an asterisk following the fundamental period.

Only the most massive main-sequence models have convective cores of appreciable size. The fractional mass of the convective core, $m_{\mathrm{cc}} / M$, is $Z$-dependent, and the post-main-sequence evolutionary tracks consequently also change with metal content. The Hertzsprung gap due to rapid core contraction prior to central hydrogen exhaustion is present only for the more massive $Z=10^{-4}$ models, although there is a suggestion of a weak gap in the $2.5 M_{\odot}, Z=10^{-6}$ evolutionary track. None of the models had a semiconvective zone during core hydrogen burning.

Following exhaustion of the central hydrogen and the formation of a shell source, all models evolve toward the red-giant region of the (luminosity, effective temperature)-plane; however, the subsequent evolutionary behavior can be of two varieties. The first type of behavior is that of classical development as a red giant: the star eventually develops a deep convective envelope and ascends the giant branch. Owing to conditions of extreme degeneracy and rapid neutrino production, the tracks of these models tend to converge in the (central density, central temperature)-plane near $\log T_{c} \simeq 7.9$. Eventually, at lumi- nosity in the range $\log L / L_{\odot} \simeq 2.5-3.0$ and effective temperature $\log T_{e} \simeq 3.62$, energy production from the triple-alpha reaction finally exceeds that lost in neutrino emission. In the least massive models, neutrino losses in the center are so large that helium ignition initially occurs off center; however, a convective core develops shortly afterward and destroys the resulting small outward composition gradient. The evolution of such stars has been well-studied in the past, and the present evolutionary tracks agree well with results of Simoda and Iben (1970), Rood (1972; Iben and Rood 1970), and Demarque and Mengel (1971, 1973).

The second type of behavior appears in more massive models which succeed in igniting helium under less degenerate conditions before reaching the tip of the red-giant branch. The members of this group tend to be pulsationally unstable, a point which is discussed in greater detail below. The transition mass $M_{\mathrm{tr}}$ between the two types is a function of composition, decreasing with decreasing $Z$ at $X=0.739$ from $1.8 M_{\odot}$ at $Z=10^{-4}$ to $1.25 M_{\odot}$ at $Z=10^{-6}$ to $1.2 M_{\odot}$ at $Z=10^{-8}$, and increasing with increasing $X$ at $Z=10^{-8}$ from $1.2 M_{\odot}$ at $X=0.739$ to $1.85 M_{\odot}$ at $X=0.9999$, where the above values were interpolated along the helium ignition line in the (central density, central temperature)-plane. The evolution of this higher-mass group is qualitatively very similar 
to the behavior of 2.0 and $3.0 M_{\odot}$ model sequences for $(X, Z)=\left(0.7,10^{-3}\right)$ of Trimble, Paczyński, and Zimmerman (1973). The $X=0.739$ models reported here have higher effective temperatures, central densities, and central temperatures on the main sequence than the $Z=10^{-3}$ models for given mass, but the luminosities are slightly lower owing to the higher initial hydrogen content. Owing to the effect of reduced metal content on the interior structure, the lower $Z$ models ignite core helium "earlier" on their evolutionary tracks than do the $Z=10^{-3}$ models.

The two sets of essentially pure hydrogen-helium models are qualitatively similar, except for a decrease in the luminosities of the extremely hydrogen-rich models by $0.4-0.5$ in the logarithm, as well as somewhat lower effective temperatures. As a consequence of the low envelope temperatures, the main-sequence 0.65 and $0.75 M_{\odot}$ models have deep convective envelopes with effective temperatures lower than during their red-giant phases. As hydrogen burning in the core proceeds, these stars evolve to slightly higher effective temperatures and their envelopes become predominately radiative at post-main-sequence ages of about 60 and 20 billion years, respectively. Subsequently, the evolutionary tracks turn toward lower effective temperatures and the envelope convective region again reaches below $m_{\mathrm{fit}}=0.9 \mathrm{M}$, resulting in a second convective envelope phase which lasts through the subsequent evolution. The $0.85 M_{\odot}$ model starts with a radiative envelope, but also develops a deep convective envelope prior to core hydrogen exhaustion, which follows by only 76 million years. The transition mass for the appearance of this convective envelope phase during core hydrogen burning must be only marginally larger than $0.85 M_{\odot}$; the transition mass for the presence of a convective envelope throughout core hydrogen burning will be near $0.6 M_{\odot}$.

The evolutionary tracks in figures $1 a-4 a$ have been used to interpolate time-constant loci for each composition which are reproduced in figures 5 and 6 . The isochrones shown correspond to ages of $6,8,10,12$, 15 , and 20 billion years. Although the zero-age main sequence is essentially identical for the three compositions with $X=0.739$, there are noticeable differences in the locations of the main-sequence turnoff, luminosities on the subgiant branch, and height of the giant branch for the same age and different $Z$. Isochrones for the very hydrogen-rich composition are more rounded at the main-sequence turnoff than the $X=0.739$ sequences. For ages of 13 and 20 billion years, the maximum red-giant mass for $X=0.739$ should be about $0.74 M_{\odot}$ and $0.67 M_{\odot}$, respectively; while for the long-lived $X=0.9999$ models, the corresponding values are $1.1 M_{\odot}$ and $1.0 M_{\odot}$.

\section{PULSATION ANALYSIS}

As indicated above, the results of the pulsation analysis of models at the principal points are summarized in the last columns of tables 1-4. Models found to be unstable in the fundamental radial mode are indicated by an asterisk following the entry of the fundamental period. The computed values of the fundamental-mode pulsation constants cover the range $0.045 \leqslant Q_{0} \leqslant 0.07$, with a mean of about 0.05 day. Typically, during the course of evolution $Q_{0}$ decreases
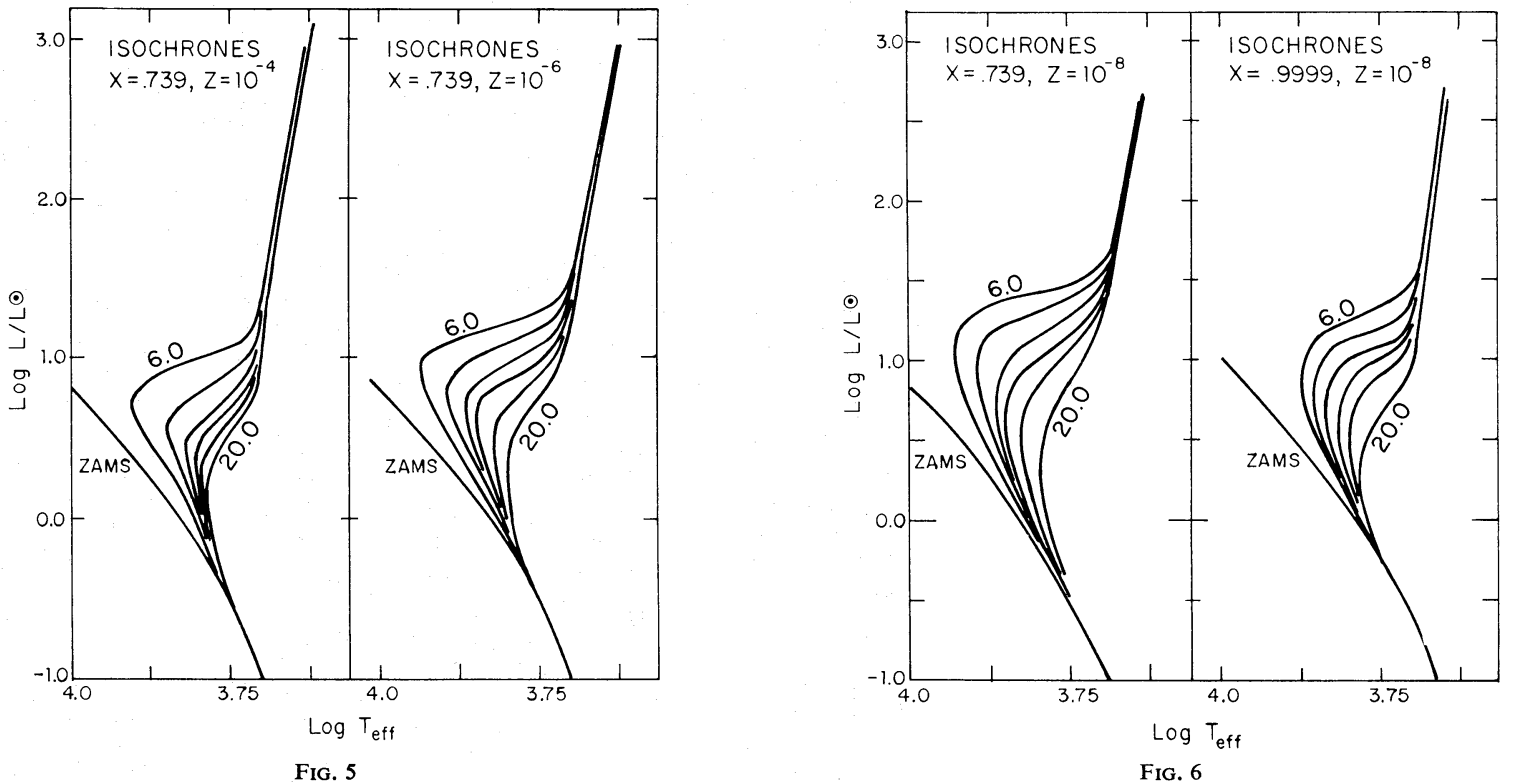

FIG. 5.- Isochrones and zero-age main sequences for $(X, Z)=\left(0.739,10^{-4}\right)$ and $\left(0.739,10^{-6}\right)$. Isochrone ages are $6,8,10,12$, 15 , and 20 billion years.

FIG. 6.-Isochrones and zero-age main sequences for $(X, Z)=\left(0.739,10^{-8}\right)$ and $\left(0.9999,10^{-8}\right)$. Isochrone ages as in fig. 5 . 
slightly from its main-sequence value until central hydrogen exhaustion, reaches a value of 0.048 at the onset of deep envelope convection, and then increases to $Q_{0} \geqslant 0.052$ at core helium ignition. There is a noticeable tendency for the lower-mass stars to have larger $Q_{0}$ at corresponding evolutionary stages than more massive stars.

Owing to destabilization both by nuclear reactions in the hydrogen-burning core and strong convection, the lowest-mass main-sequence models for each composition are pulsationally unstable. In all cases, the instability disappears near core hydrogen exhaustion. Similar behavior in the case of a $0.5 M_{\odot}$ model sequence with $(X, Z)=\left(0.90,10^{-3}\right)$ has been found by Boury and Noels (1973). The consistent results for all the above compositions suggests that a search for pulsators among very metal-poor field stars of low mass should be made. The fundamental mode periods of such stars should be about 45 minutes for essentially unevolved stars and range up to a few hours for somewhat more massive or more hydrogen-rich stars. Although a linearized calculation cannot give the surface amplitudes for the pulsations, the relative sizes of the terms of the pulsation equation (1) in the various models suggest that the amplitudes may be small. Photometry of a few late-type extreme subdwarfs could provide a check of the above prediction. In the absence of detectable pulsations, the suggestion of Boury and Noels (1973) that our treatment of convection in models of these stars is seriously inadequate would become increasingly attractive.

Pulsational instability also appears during core helium ignition in the most massive stars. These stars all have hydrogen-burning shells of moderate thickness (up to 6 percent of the total mass) and helium ignition occurs under less degenerate conditions than in lowermass stars. Neutrino losses are still high enough, however, so that there is an off-center local maximum of the luminosity in the convection core. Destabilization of these models is aided by a relatively small mass-toluminosity ratio $\left(l \sim 10^{-3}\right)$, resulting in a comparatively large value of pulsation coefficient $D \simeq+0.6$ in the region of peak core luminosity. Coefficients $A$ and $B$ have opposite sign within the convective portion of the helium-rich core $(B<0)$, which also contributes to the instability. In the radiative part of the core both are negative; however, the zones are still unstable, although to a lesser degree, and the unstable region extends into the base of the shell source. We thus have a case of the pulsation of the entire star being driven by the hydrogen-exhausted core, since the ionization zones in the envelope are only marginally unstable.

A third group of pulsationally unstable models appears among the evolutionary sequences for the very hydrogen-rich composition, the instability appearing for stars of masses 1.0-1.5 $M_{\odot}$ at core hydrogen exhaustion.

Eigensolutions for all principal point models have been inspected in detail. Main-sequence models all show a smooth variation of the amplitudes with fractional mass $m / M$, with the unstable models having significantly large relative amplitudes throughout. All models with hydrogen-exhausted cores show a smooth decrease in amplitude as fractional mass is decreased from unity to the outer boundary of the hydrogen shell source. The quasi-adiabatic luminosity eigensolution, however, possesses a strong node at or near the boundary of the helium-rich core and the shell source in all pulsationally stable models. Unstable models lack this feature entirely, or else it is only weakly present. This behavior suggests that instability may be associated with the temperature and pressure sensitivity of the opacity and neutrino loss rate in the region below the shell source even though the temperature and pressure amplitudes there are quite small.

\section{DISCUSSION}

From the results presented, it can be seen that the evolution of metal-deficient stars remains sensitive to the initial metal content down to $Z \leqslant 10^{-6}$. For stars of moderate mass, $M \geqslant 2.0 M_{\odot}$, changing $Z$ affects the convective core size and initial post-mainsequence evolution for values of $Z \geqslant 10^{-6}$. The $Z$-dependence of the interior opacity is somewhat stronger, the computed evolutionary tracks and isochrones clearly showing the effect of increased transparency in the stellar interiors as $Z$ is decreased, but the opacity sensitivity to metal content also cuts off near $Z \simeq 10^{-6}$. Increasing the initial hydrogen abundance at fixed $Z$ increases the interior opacity, due mainly to electron scattering, shifting the surface temperature and luminosity to lower values. The densities and temperatures in the hydrogen-burning regions are also shifted to lower values at corresponding evolutionary stages and, of course, the time duration of the hydrogen-burning stages increases.

These compositional effects introduce the possibility of stellar lifetimes very different from those of Population I stars. For fixed initial hydrogen content, reduction of the metal content from Population I to Population II values (e.g., from $Z \simeq 0.02$ to $Z \simeq$ $10^{-3}-10^{-4}$ ) reduces the lifetimes for low-mass stars by up to a factor of 3 . If the initial hydrogen abundance is increased, then it is possible to increase the stellar lifetime. This result suggests that the usual assumption of composition-independent stellar lifetimes in the calculation of galactic-evolution models may be a serious oversight. The situation is illustrated in figure 7 , where the lifetimes from main sequence to core helium ignition computed here are compared to the Population I lifetimes of Iben $(1965,1967 a, b)$ and to the Limber (1960) mass-lifetime relation, which has been customarily used in galactic-evolution models (cf. references of $\S \mathrm{I}$ ). If a high primordial abundance of helium, such as that resulting from a big bang, is assumed, then it is clear from figure 7 that the lifetimes of low-mass, metal-poor stars are seriously overestimated by either the Limber relation or Population I lifetimes.

The results of this study can be briefly summarized as follows:

1. The evolutionary characteristics and lifetimes of metal-deficient stars of low mass are sensitive to initial 


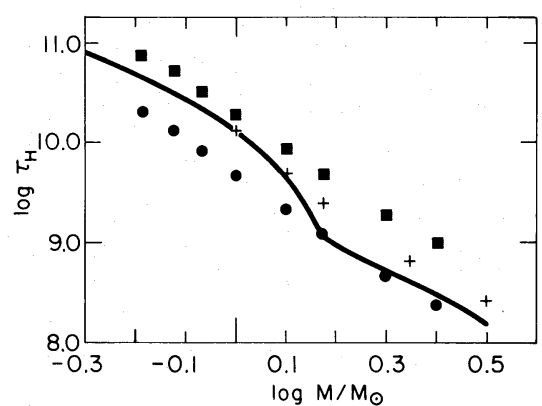

FIG. 7.-Comparison of published Population I stellar lifetimes and metal-poor stellar lifetimes presented here. Filled circles, $X=0.739$ models; filled squares, $X=0.9999$ models; crosses, models of Iben $(1965,1967 a, b)$; solid line, relation of Limber (1960).

composition. The sensitivity to metal abundance effectively ceases near $Z \simeq 10^{-6}$.

2. Such stars remain secularly stable throughout their evolution from the main sequence to core helium ignition.

3. Low-mass main-sequence stars of extremely low metal content are pulsationally unstable in the fundamental radial mode with periods near 0.67 hour owing to destabilization by both convection and nuclear burning. The instability disappears near central hydrogen exhaustion.

4. Metal-deficient stars of intermediate mass may ignite core helium before reaching the giant branch. These stars tend to be pulsationally unstable.

5. Galactic-evolution calculations using the assumption of composition-independent stellar lifetimes may be somewhat in error in their requirements on lowmass members of any initial stellar generation.

In light of the above results, it is urged that future models for the evolution of the Galaxy include composition-dependent stellar lifetimes, particularly for stars of low and intermediate mass. A search for pulsations in very-metal-poor subdwarfs is also urged.

The author wishes to express his thanks to W. B. Hubbard, Jr., B. Warner, D. L. Lambert, and C. N. Davids for many helpful discussions. Thanks are also due to the referee for suggestions which resulted in the improvement of this paper. Computation funding for this project was provided by the Computation Center of the University of Texas at Austin. This paper is based in part upon a dissertation presented to the faculty at the University of Texas at Austin.

\section{REFERENCES}

Baker, N. 1966, in Stellar Evolution, ed. R. F. Stein and A. G. W. Cameron (New York: Plenum Press), p. 333.

Bardeen, J. M., Thorne, K. S., and Meltzer, D. W. 1966, Ap. J., 145, 505.

Barnes, C. A. 1971, Adv. in Nucl. Phys., 4, 133.

Beaudet, G., Petrosian, V., and Salpeter, E. E. 1967, Ap. J., 150, 979.

Beaudet, G., Salpeter, E. E., and Silvestro, M. L. 1972, Ap. J., 174, 79.

Böhm-Vitense, E. 1958, Zs. f. Ap., 46, 108

Boury, A., and Noels, A. 1973, Astr. and Ap., 24, 255.

Christy, R. F. 1966, Ann. Rev. Astr. and Ap., 4, 353. 1967, Meth. in Comp. Phys. 7, 191.

Cox, A. N., and Stewart, J. N. 1969, Sci. Inf. Astr. Council $U S S R, 15$.

1970a, Ap. J. Suppl., 19, 261.

$1970 b$, ibid., 243.

Demarque, P., and Mengel, J. G. 1971, Ap. J., 164, 317.

-. 1973, Astr. and Ap., 22, 21.

Eggen, O. J., Lynden-Bell, D., and Sandage, A. R. 1962, Ap. J., 136, 748

Fowler, W. A. 1972, in Cosmology, Fusion and Other Matters, ed. F. Reines (Boulder, Colorado: University Press).

Fowler, W. A., Caughlan, G. R., and Zimmerman, B. A. 1967, Ann. Rev. Astr. and Ap., 5, 525.

Greenstein, J. L. 1970, Comm. Ap. and Space Phys., 2, 85.

Henyey, L. G., Forbes, J. E., and Gould, N. L. 1964, Ap. J., 139, 306.

Henyey, L. G., Wilets, L., Böhm, K. H., LeLevier, R., and Levee, R. D. 1959, Ap. J., 129, 628.

Hubbard, W. B., and Lampe, M. 1969, Ap. J. Suppl., 18, 297.

Iben, I. 1965, Ap. J., 142, 1447.
Iben, I. 1967a, Ap. J., 147, 624.

$1967 b$, ibid., p. 650.

Iben, I., and Rood, R. T. 1970, Ap. J., 159, 605.

Larson, R. B. 1972, Nature, 236, 21.

Ledoux, P., and Walraven, T. 1958, in Handbuch der Physik, ed. S. Flügge (Berlin: Springer-Verlag), Vol. 51.

Limber, D. N. 1960, Ap. J., 131, 168.

Merts, A. L., and Magee, N. H. 1972, private communication. Paczyński, B. 1969, Acta Astr., 19, 1.

- 1970, ibid., 20, 47.

Pagel, B. E. J. 1970, in Vistas in Astronomy, Vol. 12, ed. A. Beer (Oxford: Pergamon).

Quirk, W. J., and Tinsley, B. M. 1973, Ap. J., 179, 69.

Reeves, H. 1965, in Stellar Structure, ed. L. H. Aller and D. B. McLaughlin (Chicago: University of Chicago Press).

Robertson, J. W., and Faulkner, D. J. 1972, Ap. J., 171, 309.

Rood, R. T. 1972, Ap. J., 177, 681.

Schmidt, M. 1963, Ap. J., 137, 758

Schwarzschild, M., and Härm, R. 1958, Ap. J., 128, 348.

Simoda, M., and Iben, I. 1970, Ap. J. Suppl., 22, 81.

Talbot, R. J. 1973, preprint.

Talbot, R. J., and Arnett, W. D. 1971, Ap. J., 170, 409. $1973 a$, Ap.J., 186, 51 .
1973 b, ibid., p. 69 .

Trimble, V., Paczyński, B., and Zimmerman, B. A. 1973, Astr. and Ap., 25, 35.

Truran, J. W., and Cameron, A. G. W. 1971, preprint.

Truran, J. W., Hansen, C. J., and Cameron, A. G. W. 1965, Canadian J. Phys., 43, 1616.

Vitense, E. 1953, Zs. f. Ap., 32, 135.

Wagoner, R. V. 1973, Ap.J., 179, 343.

RAYMOND L. WAGNER

Department of Astronomy, BJ-20, University of Washington, Seattle, Washington 98195 